\title{
Kinematical rainbow and elastic focusing effects in atom-surface scattering
}

\author{
S. Miret-Artés \\ Instituto de Matemáticas y Física Fundamental, Consejo Superior de Investigaciones Científicas, Serrano, 123, 28006 Madrid, Spain \\ J. R. Manson \\ Department of Physics and Astronomy, Clemson University, Clemson, South Carolina 29634
}

(Received 12 January 1999)

\begin{abstract}
In this work two general anomalous singularity effects in elastic surface diffraction are reported. First, a kinematical rainbow $(\mathrm{KR})$ condition, a special case of the well-known rainbow singularity, is examined and shown to be a two-dimensional counterpart of the glory effect in gas-phase scattering. Second, an elastic focusing $(\mathrm{EF})$ effect is presented as the elastic counterpart of a general effect called inelastic focusing recently observed in the inelastic scattering of $\mathrm{He}$ atoms from a $\mathrm{NaCl}(001)$ surface. Both effects occur under conditions in which the angular positions of the diffraction channels are degenerate in pairs. In the vicinity of the KR the coupling among diffraction channels is strongly rearranged and noticeable changes in the diffraction pattern intensities are expected, whereas the EF enhances the observed intensity of only the affected diffraction peak. As an illustration of these general phenomena in surface scattering, calculations are presented demonstrating that both of these new effects should be observable for He atom scattering from stepped metal surfaces. [S0163-1829(99)14127-4]
\end{abstract}

In recent years, a number of new resonance mechanisms and focusing phenomena (scattering singularities) have been reported in the field of surface scattering. ${ }^{1-7}$ In general, all of these focusing effects are related to singularities of one or more Jacobians appearing in the theoretical expressions for the observable differential reflection coefficients. Two different sources of these effects can be distinguished. The first one occurs when the differential reflection coefficient is expressed in terms of Jacobians of some final kinematic variable with respect to an initial one. Singularities in the Jacobian (density of states) can be manipulated by varying the initial conditions of the experiment, such as the energy resolution of the incident beam, and are not directly related to the nature of the scattering potential. An example of such a singularity is inelastic focusing (IF), recently observed in the scattering of $\mathrm{He}$ atoms from the $\mathrm{NaCl}(001)$ surface. ${ }^{6}$ The second source of singularities depends on the nature of the interaction potential and arises when a classical description of the physical process leads to singularities in the corresponding Jacobians, as in the case of the classical surface rainbow. Although these singularities are general to any type of surface scattering projectiles (e.g., photons, electrons, or neutrons), a large number has been observed in atom scattering due to the recent development of very nearly monoenergetic beams of high intensity and the improvement of detection methods yielding large dynamic ranges of observed scattered intensities. Reported in this paper are two new singular effects, one of each of the above general types.

The rainbow singularity in surface scattering is well known and is associated with classical scattering from an inflection point in the surface corrugation. Here, a new rainbow singularity is examined, the kinematical rainbow (KR), which is a counterpart of the glory scattering effect observed in gas-phase scattering. Experimental evidence for this effect, supported by close-coupling calculations, is presented for $\mathrm{He}$ atom scattering from the vicinal $\mathrm{Cu}(115)$ surface.
The recently reported IF is a unique effect in general twodimensional scattering in which the entire energy spread of the incident beam is focused at special final angles and energies. ${ }^{6}$ Previously, the energy resolution of the incident beam has been considered to have little if any effect on the angular distribution of the scattered intensity. This effect was demonstrated to enhance the intensity of weak single-phonon features in inelastic time-of-flight spectra in He atom scattering from $\mathrm{NaCl}(001)$. The counterpart in elastic scattering discussed here is called elastic focusing $(\mathrm{EF})$, and its manifestation is again illustrated in the diffraction patterns for the scattering of He atoms from the (115) stepped surfaces of a $\mathrm{Cu}$ crystal.

For simplicity the theoretical development will be limited to one-dimensional crystal surfaces but the extension to two dimensions is trivial, and the general conclusions drawn remain valid. The incident angle $\theta_{i}$ is measured with respect to the surface normal (which is chosen to be the $z$ axis), and is taken to be positive. The final angle $\theta_{f}$ is positive if it is in the opposite quadrant from the incident angle and negative if it is in the same quadrant. The corrugation of the surface is represented by the function $\xi(x)$ where $x$ is the direction parallel to the surface and a normalized impact parameter is defined by $b=x / a$ with $a$ the surface unit cell length. The reciprocal lattice vector is denoted by $g_{n}=2 n \pi / a$, with $n$ being the order of the diffraction. Energies are written in units such that $\hbar^{2} / 2 m=1$.

The starting point is the kinematical conditions of conservation of parallel momentum

$$
\Delta K=k_{f} \sin \theta_{f}-k_{i} \sin \theta_{i}=g_{n},
$$

and conservation of energy

$$
\Delta E=k_{f}^{2}-k_{i}^{2},
$$


where $\mathbf{k}_{f}$ and $\mathbf{k}_{i}$ are the wave vectors of the final and initial states, respectively. For purely elastic processes $\Delta E=0$. The momentum transfer in the direction normal to the surface is

$$
\Delta k_{z}=-k_{i} \cos \theta_{i}-k_{f} \cos \theta_{f},
$$

and the $z$ component of momentum in the scattered diffraction channel corresponding to $g_{n}$ is given by

$$
k_{n z}^{2}=k_{i}^{2}-\left(k_{i} \sin \theta_{i}+g_{n}\right)^{2} .
$$

The classical reflection condition for a particle scattering from a surface is given by

$$
g_{n}+\xi_{a}^{\prime}(b) \Delta k_{z}=0,
$$

with $\xi_{a}^{\prime}(b) \equiv \xi^{\prime}(b) / a$ being the first derivative of the corrugation function with respect to $b$. Equation (5) is valid for classical scattering with $g_{n}$ replaced by an arbitrary transfer of parallel momentum $\Delta K$. The classical limit, in this case, is equivalent to having a lattice periodicity $a$ which is very large so that the $g_{n}$ are closely spaced. However, for the purposes of this paper, quantum-mechanical diffraction implies that $\Delta K=g_{n}$ and $g_{n}$ will be taken to be constant associated with a particular diffraction order. This condition implies that if Eq. (5) is differentiated with respect to the impact parameter $b$, then $\xi_{a}^{\prime \prime}\left(b_{p}\right)=0$, which is the ordinary condition for the rainbow singularity associated with the inflection points of the corrugation function at $b=b_{p}$.

It is convenient to develop the diffraction intensities in terms of the semiclassical formalism of Maisel, Merrill, and Miller. ${ }^{8}$ The classical deflection function $\theta_{f}(b)$ is defined by ${ }^{9,10}$

$$
\theta_{f}(b)=\theta_{i} \pm 2|\theta|=\theta_{i} \pm 2\left|\tan ^{-1} \xi_{a}^{\prime}(b)\right|,
$$

where the $(+)$ sign is used when $\theta<0$ and the $(-)$ sign otherwise. Here, $\theta$ is measured following the convention used for the final angle. Similarly, the classical diffraction order function is defined by ${ }^{8-10}$

$$
\begin{aligned}
J\left(b ; k_{i}, \theta_{i}\right) & =\frac{a k_{i}}{2 \pi}\left[\sin \theta_{f}(b)-\sin \theta_{i}\right] \\
& =\frac{a k_{i}}{\pi} \frac{\left|\xi_{a}^{\prime}(b)\right|}{1+\xi_{a}^{\prime}(b)^{2}}\left[ \pm \cos \theta_{i}-\left|\xi_{a}^{\prime}(b)\right| \sin \theta_{i}\right],
\end{aligned}
$$

where the diffraction condition is obtained when $J\left(b ; k_{i}, \theta_{i}\right)$ takes on integer values, or when

$$
g_{n}=\frac{2 \pi}{a} J\left(b ; k_{i}, \theta_{i}\right) .
$$

The semiclassical diffraction intensity is then given by ${ }^{8}$

$$
I_{J}\left(k_{i}, \theta_{i}\right)=\sum_{j}\left|\frac{d J\left(b_{j} ; k_{i}, \theta_{i}\right)}{d b}\right|^{-1},
$$

where the sum is over all values of the impact parameter $b_{j}$ that contribute to a given integer value, $J\left(b ; k_{i} ; \theta_{i}\right)=n$.

Surface rainbows and other types of singularities occur when the derivative $d J\left(b_{j} ; k_{i}, \theta_{i}\right) / d b$ vanishes. In order to clearly exhibit such singularities, it is usually more conve- nient to express the intensity $I_{J}\left(k_{i}, \theta_{i}\right)$ in terms of the measurable physical variables through Jacobian relations. For example, the ordinary rainbow is readily exhibited by rewriting the derivative in Eq. (9) as $^{9}$

$$
\frac{d J\left(b_{j} ; k_{i}, \theta_{i}\right)}{d b}=\frac{d J\left(b_{j} ; k_{i}, \theta_{i}\right)}{d \theta_{f}} \frac{d \theta_{f}(b)}{d b} .
$$

Singularities in the intensity of Eq. (9) can arise when either of the two derivatives in the right-hand side of Eq. (10) vanishes. As has been shown elsewhere, ${ }^{9,10}$ zeros of the first factor, $d J / d \theta_{f}=0$, are responsible for the so-called skipping singularity and also lead to the threshold singularity. The phenomenon of skipping of atoms also has its counterpart in the skipping of electrons. Electrons close to the surface of a metal, and in a magnetic field applied parallel to the surface, bounce many times. ${ }^{11}$ Zeros of the second factor on the right-hand side of Eq. (10), $d \theta_{f} / d b=0$, correspond to the well-known classical surface rainbows. ${ }^{12}$ For example, the derivative is readily evaluated from Eq. (6) and leads to $\xi_{a}^{\prime \prime}\left(b_{p}\right)=0$, which implies that a classical rainbow feature occurs whenever the classical scattering angle of Eq. (6) corresponds to an inflection point in the surface corrugation. In the quantum-mechanical case, in which the elastic scattering from an ordered surface consists only of distinct diffraction beams, the rainbow does not appear as a singularity but results in a greatly enhanced diffraction beam or group of diffraction beams in the neighborhood of the rainbow angle. Since the scattering is unitary and all diffraction beams are coupled, this rainbow enhancement will also result in a rearrangement of the intensities in all diffraction beams, thus the rainbow feature is associated with the entire diffraction pattern and not just the diffraction peaks near the rainbow angle. In general, the derivative $d \theta_{f} / d b$ appearing in Eq. (10) is replaced by a Jacobian function, and the relations between the topology of the surface and the zeros of this Jacobian have been expressed in terms of caustics. ${ }^{13}$

In this paper we wish to consider a special type of rainbow singularity that is subject to the additional constraint of the classical reflection condition of Eq. (5). An alternative expression involving the reciprocal lattice vector $g_{n}$ and the corrugation slope $\xi_{a}^{\prime}(b)$ is obtained from Eqs. (1), (3), and (5) and is

$$
\tan \theta_{f}=\frac{g_{n}+k_{i} \sin \theta_{i}}{g_{n} / \xi_{a}^{\prime}-k_{i} \cos \theta_{i}} .
$$

Then the rainbow singularity condition appearing in Eq. (10) is

$$
\frac{d \theta_{f}}{d b}=\frac{\xi_{a}^{\prime \prime}\left(b_{p}\right)}{\xi_{a}^{\prime}\left(b_{p}\right)^{2}} \frac{g_{n}\left[g_{n}+k_{i} \sin \theta_{i}\right]}{k_{i}^{2}+\bar{E}}=0,
$$

with

$$
\bar{E}=g_{n}^{2}+\frac{g_{n}^{2}}{\xi_{a}^{\prime}\left(b_{p}\right)}+2 k_{i} g_{n}\left(\sin \theta_{i}-\frac{\cos \theta_{i}}{\xi_{a}^{\prime}\left(b_{p}\right)}\right) .
$$

Equation (12) is a new condition for rainbow singularities, and its numerator is the product of three factors each of which can vanish. The first of these factors is the second 
derivative of the corrugation function whose vanishing is the cause of the ordinary surface rainbow. However, since Eq. (11) is obtained from Eq. (5), which in turn implies $\xi_{a}^{\prime \prime}\left(b_{p}\right)$ $=0$, it is seen that the ordinary surface rainbow condition will always apply when Eq. (12) is satisfied. The third factor in the numerator of Eq. (12) vanishes when

$$
g_{n}=-k_{i} \sin \theta_{i}<0,
$$

which according to Eq. (1) implies that $\theta_{f}=0$ is a Bragg angle. Thus we have the result that if the diffraction beam associated with the reciprocal lattice vector $g_{n}$ is at an angle satisfying the rainbow condition $\xi_{a}^{\prime \prime}\left(b_{p}\right)=0$ and this angle is also normal to the surface $\theta_{f}=0$, then this corresponds to a "superrainbow" condition in which two factors in the numerator of Eq. (12) are vanishing. This new condition is called the kinematical rainbow. By substituting Eq. (14) into Eq. (13), we have that $\bar{E}=0$. It is also noticed that the second factor in the numerator of Eq. (12), $g_{n} / \xi_{a}^{\prime}\left(b_{p}\right),{ }^{2}$ which through Eq. (5) can be replaced by $\Delta k_{z}^{2} / g_{n}$, does not go to zero for nongrazing incident angles.

The KR condition of Eq. (14) is reminiscent of the glory (or zero-deflection) effect in gas-phase scattering. ${ }^{14}$ In glory scattering certain solid angles become very small and result in enhanced intensity due to the interference between the unscattered particles and those going in the forward- and backward-scattering directions. In our case, the impact parameters $b_{j}$ from Eq. (9) contributing to a zero Bragg angle will have corresponding classical trajectories whose amplitudes interfere to give rise to some oscillation in the diffraction pattern. The main difference is that in this context this singularity is always accompanied by the rainbow condition.

It is interesting to note that under KR conditions with $\theta_{f}$ $=0$ the normal momentum of the corresponding diffraction beam is identical with the magnitude of the incident momentum, $k_{n z}=k_{i}$. Furthermore, the angular positions of all other diffraction peaks are degenerate in pairs in the sense that they have the same $z$ component of the wave vector $k_{n z}$. That is, if the $g_{n}$ peak is at KR, the angular positions of the $g_{n-m}$ and $g_{n+m}$ peaks have equal magnitude but opposite signs (alternatively stated, the polar angles with respect to the surface normal of the $g_{n-m}$ and $g_{n+m}$ diffraction peaks are identical). Such a degeneracy was reported in Ref. 15 but it was not associated with a new singularity. An additional aspect of this singularity is that the diffraction channel displaying $\mathrm{KR}$ is the lowest (for negative $n$ values) diffraction channel accessible classically for the given surface corrugation. That means that the remaining smaller (negative $n$ ) diffraction channels allowed energetically are forbidden classically and only complex trajectories contribute to those diffraction intensities.

The KR conditions readily can be realized in experimental configurations, and we would like to offer evidence that it could be observed in the scattering of He atoms from stepped copper surfaces, such as was done in the early experiments of Gorse et al. ${ }^{16}$ For $\mathrm{Cu}(115)$ the surface unit cell dimension is $a=6.625 \AA$ and for an incident momentum $k_{i}$ $=10.98 \AA^{-1}\left(E_{i}=63 \mathrm{meV}\right)$ the condition (14) together with a final angle $\theta_{f}=0$ are satisfied by the $n=-7$ diffraction beam at an incident angle $\theta_{i}=37.11^{\circ}$.

Figure 1 shows the results of close-coupling calculations

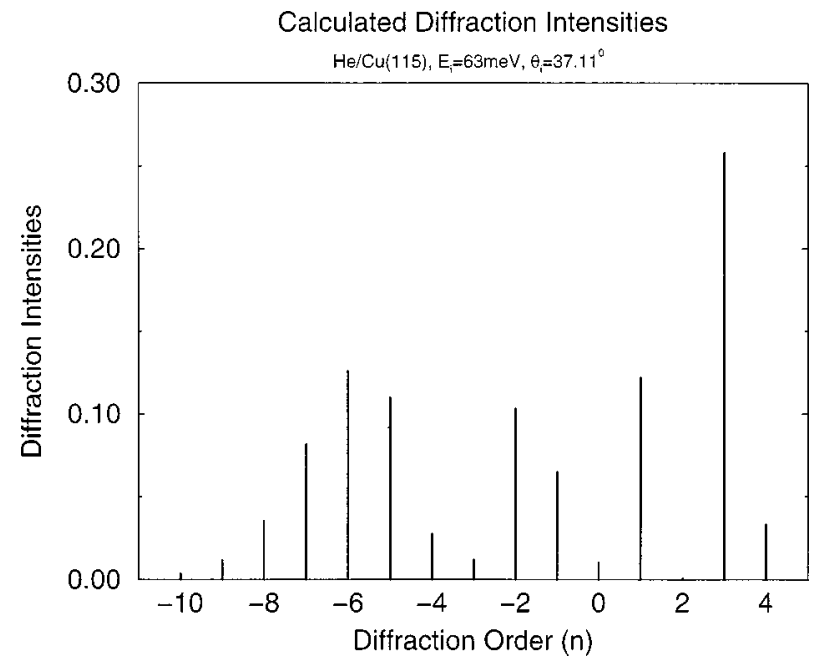

FIG. 1. Close-coupling calculation intensities plotted against the diffraction order $n$ for an incident beam of He atoms scattering from a $\mathrm{Cu}(115)$ stepped surface. The incident energy is $63 \mathrm{meV}$ and the incident angle is $\theta_{i}=37.11^{\circ}$.

of the diffraction intensities for these incident conditions for a potential model that has been previously shown to give a good match to the measured experimental data for this same surface $^{17}$ (unfortunately, there have been no reported experimental measurements at this incident angle and energy). The model consists of a Morse potential with a corrugated repulsive term. ${ }^{17}$ The stiffness parameter is $\beta=1.05 \AA^{-1}$, the well depth is $D=6.35 \mathrm{meV}$, and the stepped corrugation is explained in Ref. 17. The calculated diffraction pattern is characterized by a large rainbow peak from the step terrace at $n=3$ and a smaller, broader distribution of rainbow peaks from the step face extending from $n=-5$ to -8 that could be attributed to a glory oscillation. There are supernumerary rainbow features between these two classical rainbows. The $n=3$ terrace rainbow peak is exceptionally large with total intensity fraction of 0.258 .

The large number of peaks involved in the step-face rainbow feature in Fig. 1 and the large intensity of the $n=-7$ peak at $\theta_{f}=0^{\circ}$ are due to the kinematical rainbow. The source of the KR under these conditions is evident from Fig. 2 where the classical deflection function of Eq. (6) is shown for the same potential used in Fig. 1. Each of the four curves plotted in Fig. 2 shows the final angle for classical scattering as a function of the impact parameter $b$ for four given incident angles and for each case a total of 1000 classical trajectories were run. The incident angles correspond to the condition of Eq. (14) for the $n=-2,-4,-7$, and -8 diffraction peaks, as marked. Only the $n=-7$ diffraction peak has an inflection point at the Bragg angle $\phi_{f}=0$, as evidenced by the minimum of the classical deflection curve, which is also necessary to fulfill the conditions for KR. Furthermore, whenever a Bragg angle is tangent to one of the extrema of the classical deflection function we can speak of quantum rainbows and, in this sense, the $\mathrm{KR}$ is therefore a quantum rainbow. Thus it is clear that the special combination of conditions necessary for KR can readily be found in actual experimental situations.

We would now like to discuss a different type of singular behavior that can occur in combination with the classical 


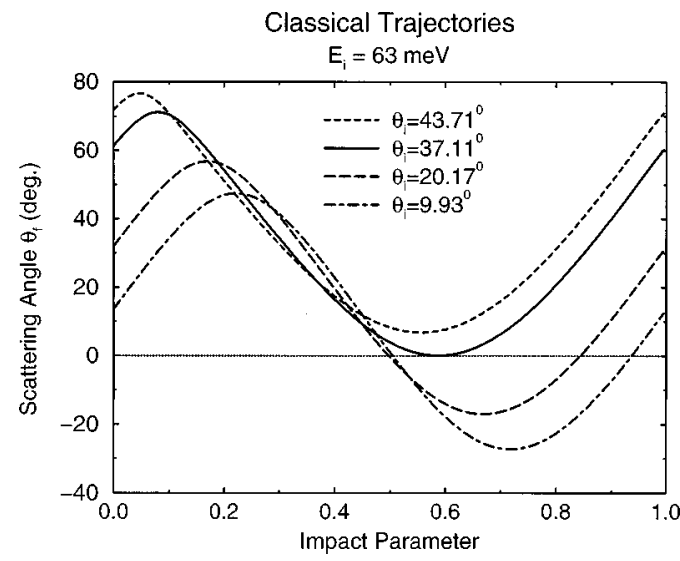

FIG. 2. Final scattering angle plotted against the impact parameter $b$ calculated using the same potential as for the close-coupled calculations of Fig. 1 with incident energy $E_{i}=63 \mathrm{meV}$. The four curves shown are for the incident angles $\theta_{i}=9.93^{\circ}, 20.17^{\circ}, 37.11^{\circ}$, and $43.71^{\circ}$, which correspond to possible KR conditions from Eq. (14) for the diffraction peaks of order $n=-2,-4,-7$, and -8 , respectively. Only the angle $\theta_{i}=37.11^{\circ}$ is a true KR because this curve also displays a concatenation at the KR (Bragg) angle of $\theta_{f}$ $=0$ for the $n=-7$ peak.

rainbow. This type of singularity does not depend on the interaction potential, instead it is driven by the initial characteristics of the experiment. Under normal experimental conditions there is always a spread of energies and angles in the incident beam. The differential reflection coefficient, which gives the fraction of particles scattered into the small energy acceptance range and into the small angular acceptance of the detector, can be written as ${ }^{6}$

$$
\frac{d R\left(\mathbf{k}_{f}, \mathbf{k}_{i}\right)}{d E_{f} d \theta_{f}}=\frac{d R\left(\mathbf{k}_{f}, \mathbf{k}_{i}\right)}{d E_{f} d k_{i}} \frac{d k_{i}}{d \theta_{f}} .
$$

In inelastic scattering, there are conditions in which the density of states (Jacobian) $d k_{i} / d \theta_{f}$ becomes large, and this effect can be used to enhance the intensity of selected inelastic features. Physically what happens is that all of the energies present in the energy width of the incident beam are focused on the chosen inelastic feature. This inelastic focusing, or IF effect, has been shown to enhance features on the Rayleigh phonon dispersion curve in $\mathrm{He}$ atom scattering from $\mathrm{NaCl}^{6}{ }^{6}$ Here our purpose is to investigate the physical implications of this focusing effect in purely elastic scattering, and this process will be called elastic focusing (EF). The importance of the Jacobian $d k_{i} / d \theta_{f}$ (or its inverse $d \theta_{f} / d k_{i}$ ) in elastic surface scattering was noticed earlier ${ }^{8}$ but the possibility that it could lead to observable singularities apparently was not recognized.

The Jacobian derivative can readily be calculated from Eq. (11) and the result is

$$
\frac{d \theta_{f}}{d k_{i}}=\frac{g_{n}\left[\cos \theta_{i}+\sin \theta_{i} / \xi_{a}^{\prime}(b)\right]}{k_{i}^{2}+\bar{E}}=0 .
$$

The numerator has two factors and their vanishing corresponds to the possible sources of this new singularity. The first factor $g_{n}=0$ corresponds to the trivial case of the specular beam that is not broadened by the energy spread of the

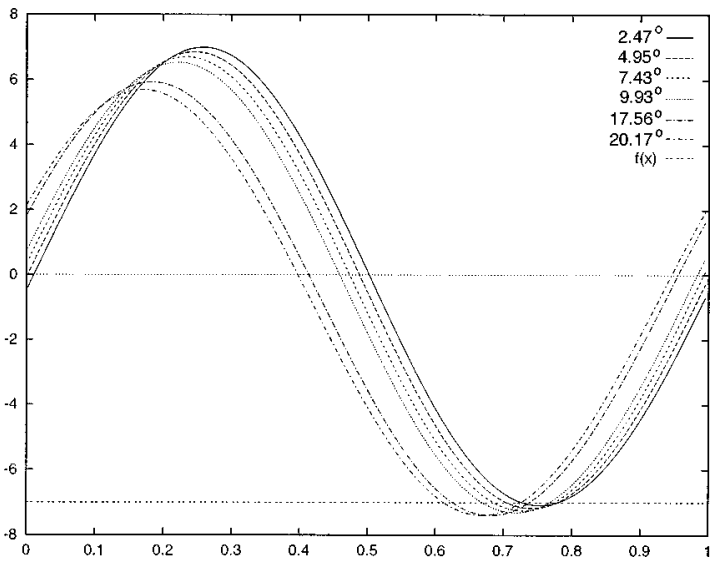

FIG. 3. The classical diffraction order function $J(b)$ of Eq. (7) plotted versus the impact parameter $b$ using the same potential for the close-coupled calculations for $\mathrm{He}$ atom scattering from $\mathrm{Cu}(115)$ of Fig. 1. As in Fig. $1 E_{i}=63 \mathrm{meV}$. The six curves displayed are for the incident angles of $\theta_{i}=2.47^{\circ}, 4.95^{\circ}, 7.43^{\circ}, 9.93^{\circ}, 17.56^{\circ}$, and $20.17^{\circ}$. To guide the eye, the horizontal lines corresponding to $J$ $=0$ and -7 are also displayed.

incident beam. Clearly, the specular scattering could be considered as a special case of EF. The other factor in the numerator when set to zero leads to

$$
\tan \theta_{i}=-\xi_{a}^{\prime}(b) \geqslant 0,
$$

or from Eq. (6)

$$
\theta_{f}=-\theta_{i},
$$

and according to Eq. (1) this singular condition will be observable only in the diffraction peak whose reciprocal lattice vector is given by

$$
g_{n}=-2 k_{i} \sin \theta_{i}<0 .
$$

The implications of Eq. (19) are very interesting. First, a diffraction peak that is simultaneously under a rainbow condition [due to Eq. (5)] and reflected backward in a direction near that of the incident beam will experience an additional enhancement due to the elastic focusing effect. The EF can consequently be used to enhance the apparent intensity of selected diffraction peaks under these unique conditions. Second, the EF is a special case under which a large portion of the energies in the energy spread of the incident beam is focused onto the same diffraction angle and the corresponding peak would be narrower and sharper than the rest of the diffraction peaks. Third, the EF does not affect the coupling with the other diffraction beams and hence causes no rearrangement of diffraction intensities. The corresponding enhancement is of kinematical nature and cannot be estimated from a unitary theory under the assumption of an incident monoenergetic plane wave. These properties are general and do not depend on the nature of the diffracting particle. Finally, it is again interesting to note that the EF occurs under conditions in which the polar angles of all diffraction peaks are degenerate in pairs, similar to the case for KR but for different angular positions. This is immediately obvious upon noting that the EF peak with reciprocal lattice vector $g_{n}$ has the same polar angle as the specular peak, as is clear from Eq. (18). Consequently, by symmetry the $g_{m}$ diffraction 


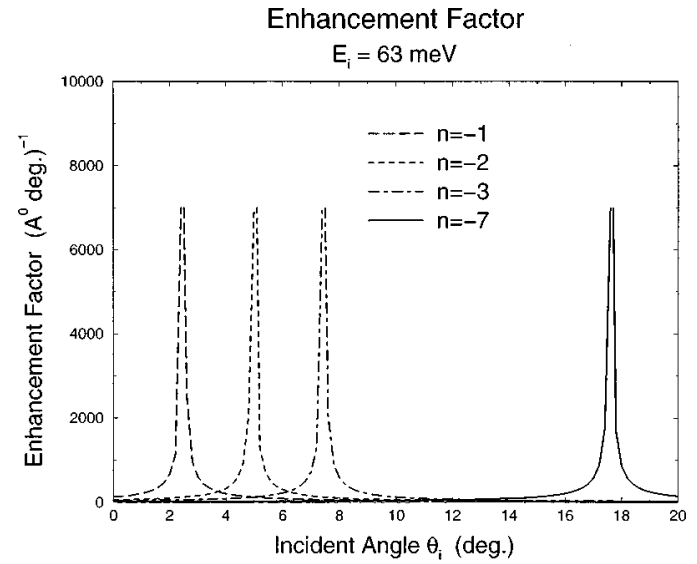

FIG. 4. The Jacobian derivative $d k_{i} / d \theta_{f}$ from Eq. (16) plotted versus incident angle for the same case of He atom scattering from $\mathrm{Cu}(115)$ as in Fig. 1 with $E_{i}=63 \mathrm{meV}$. From left to right the peaks correspond to diffraction peaks of order $n=-1,-2,-3$, and -7 as discussed in the text. This derivative can be regarded as a classical enhancement factor and it diverges at the diffraction angle for the peak at the EF condition of Eq. (19).

peak will have the same polar angle as the $g_{n-m}$ peak (or, in the convention used here, $\left.\theta_{m}=-\theta_{n-m}\right)$. Furthermore, at EF conditions, we have again that $\bar{E}=0$.

As shown above for the KR singularity, the EF can also be easily realized experimentally. Considering again the case of $\mathrm{He}$ atom scattering from $\mathrm{Cu}(115)$ at $E_{i}=63 \mathrm{meV}$ discussed above in connection with Figs. 1 and 2, one can readily determine the following series of EF singularities from Eq. (19): $n=-1$ at $\theta_{i}=2.47^{\circ} ; n=-2$ at $\theta_{i}=4.95^{\circ}$ (also a potential KR for $n=-1$ ); $n=-3$ at $\theta_{i}=7.43^{\circ} ; n$ $=-4$ at $\theta_{i}=9.93^{\circ}$ (also a potential KR for $n=-2$ ); etc. For each of these special angles we plot in Fig. 3 the classical diffraction order function of Eq. (7) versus the impact parameter $b$, where each curve is obtained from running about 1000 trajectories. As seen in Fig. 3, for all of the six EF's analyzed, their diffraction order functions display a minimum very near to the diffraction channel $n=-7$, which is the lowest diffraction channel classically accessible at $E_{i}$ $=63 \mathrm{meV}$. Obviously, only for $\theta_{i}=0$, we have a true quantum rainbow condition in the $(n=-7)$ channel representing a trivial case for EF. It is also of interest to calculate from Eq. (16) the Jacobian enhancement factor $d k_{i} / d \theta_{f}$ appearing in Eq. (15), and this is shown in Fig. 4. The enhancement is seen to be quite significant and is still large one or two degrees away from the angle $\theta_{f}=-\theta_{i}$, which should make it possible to measure this effect with a detector placed close to the incident beam.

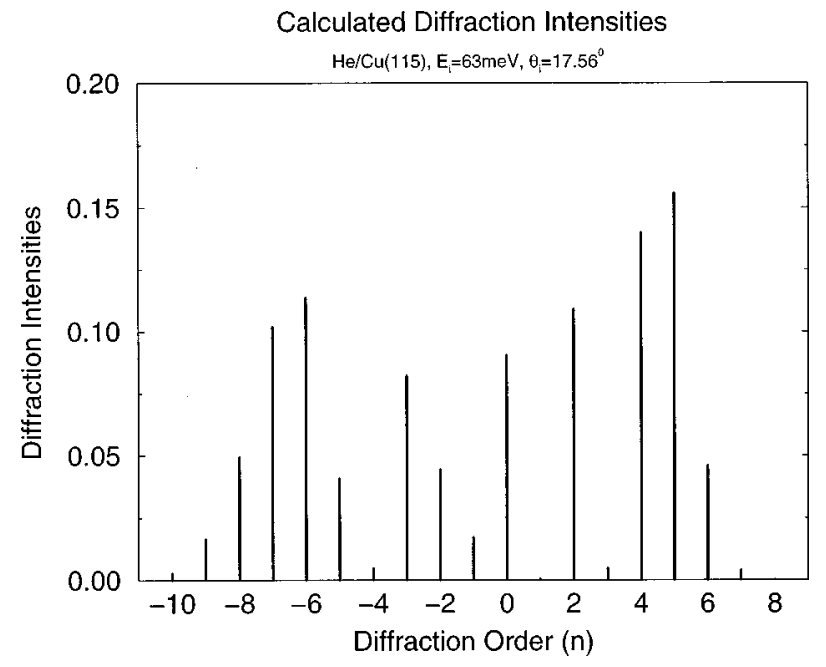

FIG. 5. Close-coupling calculations as in Fig. 1 for $\mathrm{He}$ atom scattering from the $\mathrm{Cu}(115)$ surface with incident energy $E_{i}$ $=63 \mathrm{meV}$. The incident angle is $\theta_{i}=17.56^{\circ}$.

Of particular interest is the incident angle $\theta_{i}=17.56^{\circ}$, which satisfies the EF condition of Eq. (19) for the $n=-7$ diffraction peak. The close-coupling calculation for this case, corresponding to the same potential as in Fig. 1 is shown in Fig. 5. As in Fig. 1 the diffraction pattern consists of two main classical rainbows, one due to the step terrace at the $n=4$ and 5 peak positions and one from the step face at the $n=-6$ and -7 peaks. The peaks shown in Fig. 5 are not broadened since they were calculated under the assumption of an incident monoenergetic plane wave. However, under actual experimental conditions in which the incident beam has energy and angular broadening, the $n=-7$ peak, compared to the surrounding peaks, would have a smaller width and enhanced maximum intensity due to the elastic focusing.

We have demonstrated two new singular features associated with surface diffraction called kinematical rainbow and elastic focusing. These are general features of twodimensional diffraction, and we have presented possible examples of both arising from quantum-mechanical scattering of He from stepped metal surfaces. Both of these effects cause anomalous behavior of the diffraction peaks and these anomalies can be used to obtain unique physical information. The KR gives very precise information on the position of the inflection points of the surface corrugation, and the EF allows observation of nonspecular diffraction peaks under conditions in which they are not broadened by the energy spread of the incident beam.

This work has been supported in part by NSF Grant No. DMR 9726229 and by the DGICYT (Spain) under Contract No. PB95-0071.
${ }^{1}$ G. Benedek and S. Miret-Artés, Surf. Sci. Lett. 339, L935 (1995).

${ }^{2}$ S. Miret-Artés, Surf. Sci. Lett. 366, L735 (1996).

${ }^{3}$ G. Benedek, R. Gerlach, A. Glebov, G. Lange, S. Miret-Artés, J. G. Skofronick, and J. P. Toennies, Phys. Rev. B 53, 11211 (1996).

${ }^{4}$ A. Glebov, J. R. Manson, J. G. Skofronick, and J. P. Toennies, Phys. Rev. Lett. 78, 1508 (1997).
${ }^{5}$ M. F. Bertino, S. Miret-Artés, and J. P. Toennies, Phys. Rev. B 56, 9964 (1997).

${ }^{6}$ A. Glebov, J. R. Manson, S. Miret-Artés, J. G. Skofronick, and J. P. Toennies, Phys. Rev. B 57, R9455 (1998).

${ }^{7}$ M. F. Bertino, S. Miret-Artés, and J. P. Toennies, Chem. Phys. Lett. 287, 663 (1998). 
${ }^{8}$ I. Maisel, R. P. Merrill, and W. H. Miller, J. Chem. Phys. 64, 45 (1976).

${ }^{9}$ S. Miret-Artés, J. Margalef-Roig, R. Guantes, F. Borondo, and C. Jaffé, Phys. Rev. B 54, 10397 (1996).

${ }^{10}$ R. Guantes, F. Borondo, J. Margalef-Roig, S. Miret-Artés, and J. R. Manson, Surf. Sci. Lett. 375, L379 (1997).

${ }^{11}$ I. M. Lifshitz and A. M. Kosevich, Zh. Eksp. Teor. Fiz. 29, 743 (1956) [Sov. Phys. JETP 2, 646 (1956)]; M. S. Khaikin, ibid. 39, 212 (1961) [ibid. 12, 152 (1961)]; J. W. Nee and R. E. Prange, Phys. Lett. 25A, 582 (1967).
${ }^{12}$ J. D. McClure, J. Chem. Phys. 52, 2712 (1970).

${ }^{13}$ M. V. Berry, J. Phys. A 8, 566 (1975).

${ }^{14}$ M. S. Child, in Molecular Collision Theory (Academic, London, 1974).

${ }^{15}$ M. I. Hernández, O. Roncero, S. Miret-Artés, P. Villarreal, and G. Delgado-Barrio, J. Chem. Phys. 90, 3823 (1989).

${ }^{16}$ D. Gorse, B. Salanon, F. Fabre, A. Kara, J. Perreau, G. Armand, and J. Lapujoulade, Surf. Sci. 147, 611 (1984).

${ }^{17}$ S. Miret-Artés, J. P. Toennies, and G. Witte, Phys. Rev. B 54, 5881 (1996). 